\title{
Quality assurance challenges for development of Crew Escape System Motors
}

\author{
Suvendu Jana*, S. M. Sreedhar, R. Krishnakumar, R. Vinod Kumar and \\ S. Saratchandran \\ Quality Assurance and Reliability - Fabrication Group, System Reliability Entity, Vikram Sarabhai Space Centre, \\ Thiruvananthapuram 695 022, India
}

\begin{abstract}
Crew Escape System (CES) motors for Human Rated Launch Vehicle (HRLV) have been newly designed. These solid motors have special nozzle configuration. The development of these motors was a challenging task due to stringent design specification and intricate nozzle configuration. This paper emphasizes the fabrication challenges encountered during realization of CES hardware, details of process and quality improvement done, to overcome the challenges faced during fabrication and to achieve the stringent geometrical requirements and dimensional accuracies as specified by designer.
\end{abstract}

Keywords: Crew Escape System, Escape Motors, launch vehicle, quality assurance.

\section{Introduction}

CREW Escape system (CES) is one of the most critical subsystems in a human-rated launch vehicle. Human Spaceflight Programme (HSP) requires CES motors capable of detaching and propelling the Crew Module (CM) away from the launch vehicle within the shortest time in case of any malfunctioning of the transport vehicle, or an abort during the initial regime of flight. CES of HSP consists of four types of CES motors (Figure 1), viz. Low-altitude Escape Motor (LEM), High-altitude Escape Motor (HEM), Jettisoning Motor (CJM) and Pitch Control Motor (PCM). These solid motors are newly designed and have special nozzle configuration, viz. LEM has four of reverse-flow nozzles, while CJM and HEM have nozzle with scarfed divergent configuration. Being a developmental hardware involving new design configurations, extensive care was taken to finalize the quality control plan by incorporating mandatory inspection stages, requirement and qualification procedure of drill jigs, tooling fixture and templates. Specific requirements of non-destructive testing (NDT) at different stages with acceptance criteria, welding procedure specification (WPS), qualification of special processes, identification of functionally critical dimension (FCD) inspection stages,

\footnotetext{
*For correspondence. (e-mail: suvendu_jana@vssc.gov.in)
}

storage protocols and first-off qualification guideline were implemented at various work centres from the beginning of hardware fabrication to ensure quality of the hardware. However, a few fabrication challenges were encountered during developmental phase of these hardware to meet the stringent requirements stipulated by designer. First-off hardware of CES motors was validated through successful proof pressure test (PPT). Subsequently, qualification of all CES motors has been completed through successful static tests and Pad abort test (PAT).

The major challenges encountered during realization and qualification phase of these hardware are the following:

(1) Realization and first-off qualification of reverseflow nozzle of LEM.

(2) Realization and first-off qualification of HEM scarfed divergent aft end hardware.

(3) Occurrence of high strains in motors during PPT due to profile error near the welds.

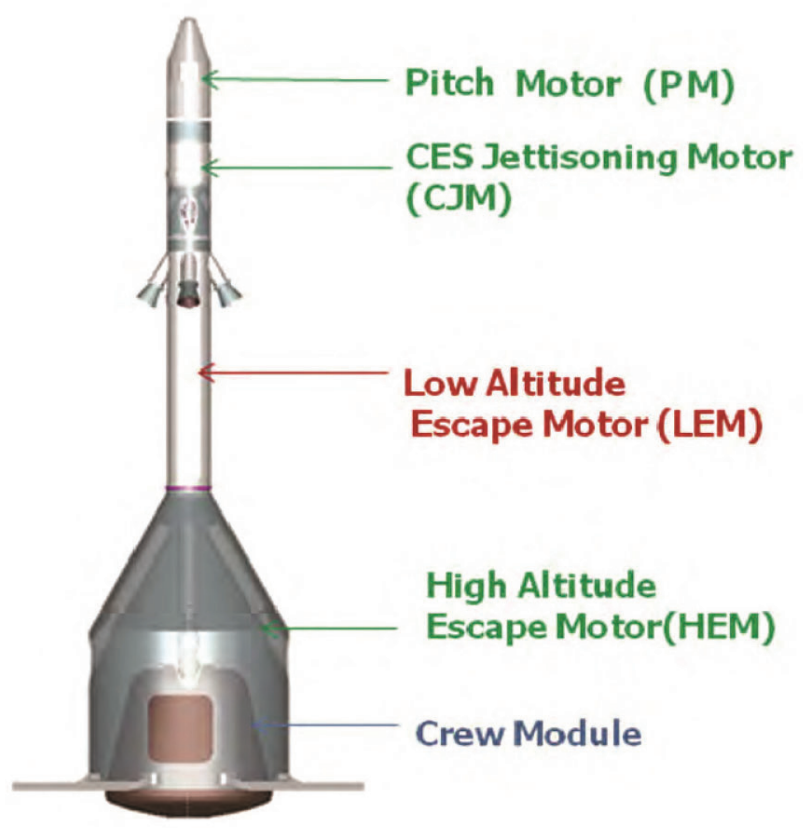

Figure 1. Crew Escape System (CES) solid motors. 


\section{Realization and first-off qualification of reverse nozzle of LEM}

LEM is one among the four solid motors in CES, whose function is to take the latter with $\mathrm{CM}$ away from the launch vehicle during emergency condition. LEM is designed with a unique configuration of a convergent hardware with four reversible nozzles mounted as reverse-flow nozzles (Figure 2). LEM nozzle consists of three components, viz. nozzle convergent (NC), end nozzle dome and nozzle skirt ring. $\mathrm{NC}$ was realized from the single forging without any welds. Forging route was adopted over welding route, to minimize the distortion at post-weld stage.

The nozzle configuration involves varying the inside profile with a cylinder joining part of a sphere in smooth contour and with four orthogonal openings with an orientation accuracy of $\pm 5^{\prime}$. This requires computer numerical control (CNC) machining of the nozzle. Figure 3 shows the critical parameters to be achieved on the nozzle.

Complete machining of intricate nozzle geometry of LEM NC required as many as 16 machining stages. All process stage setting requirements and machining operations were critically planned and finalized. Fixture and drill jigs required for various stage operations were also qualified. A trial Mild Steel (MS) nozzle convergent hardware was realized as first off and interface dimension was inspected by co-ordinate measuring machine (CMM), to validate the above fabrication process and tooling.

The most critical machining operations are highlighted below.

\section{Boring of nozzle bosses at $0^{\circ}, 90^{\circ}, 180^{\circ}$ and $270^{\circ}$ orientation}

Horizontal boring machine is used for this operation. Special qualified fixture for horizontal setting of job is made and used. Reference face and holes are provided on the fixture to dial and set it with reference to the spindle axis. Once dialling is done, the component is loaded on the fixture with the main axis of the central cylinder (which forms the NC) in horizontal orientation and aligned with the reference line already marked at the previous stage on the bottom face of $\mathrm{NC}$ at $180^{\circ}$ opposite location (Figure 4).

The internal diameter is also dialled and job is now set and clamped with strap ring. The reference line is then transferred to the two nozzle flanges on either side. This setting helps in machining the nozzle bores on either side with reference to the central axis of the job by rotating it about the vertical axis of the table from $0^{\circ}$ to $+31^{\circ}$ on the rotary table (Figure 4).

The spindle axis is moved to the specified reference distance and the nozzle bore to the required size after dialling the face of the nozzle flange. After boring, axis lines are also marked on the outside diameter (OD) of the flange with reference to the finished boring axis at $0^{\circ}$, $90^{\circ}, 180^{\circ}$ and $270^{\circ}$. The flange holes are drilled and tapping by first tap done in the same set-up using qualified drill jig. Once the nozzle bore is completed, the job is rotated back to reference and further rotated from $0^{\circ}$ to $-31^{\circ}$ for boring the second nozzle bore in the same setup, repeating the same procedure for boring the second nozzle boss. Once boring of the two nozzles is completed, the clamps are released and job rotated by $90^{\circ}$ about the horizontal axis, repeating the same procedure to generate the bores of the other two nozzles. This fabrication procedure ensures that the opposite nozzles are perfectly aligned at the same horizontal plane.

Mandatory stage inspection points were identified and in-process surveillance provided to ensure that the hardware meets the intended requirements. The specified angular orientations were measured and observed in the component as shown in Box 1.

This procedure has helped maintain the angular orientation of the nozzles accurately.

\section{Achieving spherical dome profile with control in thickness}

The nozzle end dome is welded with convergent hardware to form the nozzle (Figure 5). According to design requirement, internal dome profile at every $25 \mathrm{~mm}$ along the axis of the nozzle end dome is to be maintained and inspected. Profile measurement at the dome inside surface, which is around $1.2 \mathrm{~m}$ away from nozzle entry face, is not possible with conventional equipment due to inaccessibility. Dome profile is ensured by the process at prewelding stage according to the required specification along with $3 \mathrm{~mm}$ allowance on inside diameter (ID)

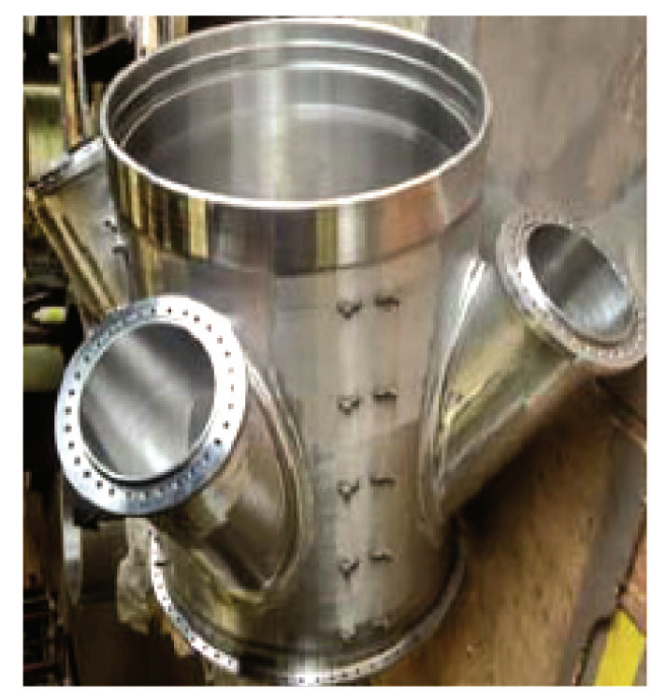

Figure 2. Nozzle convergent assembly. 


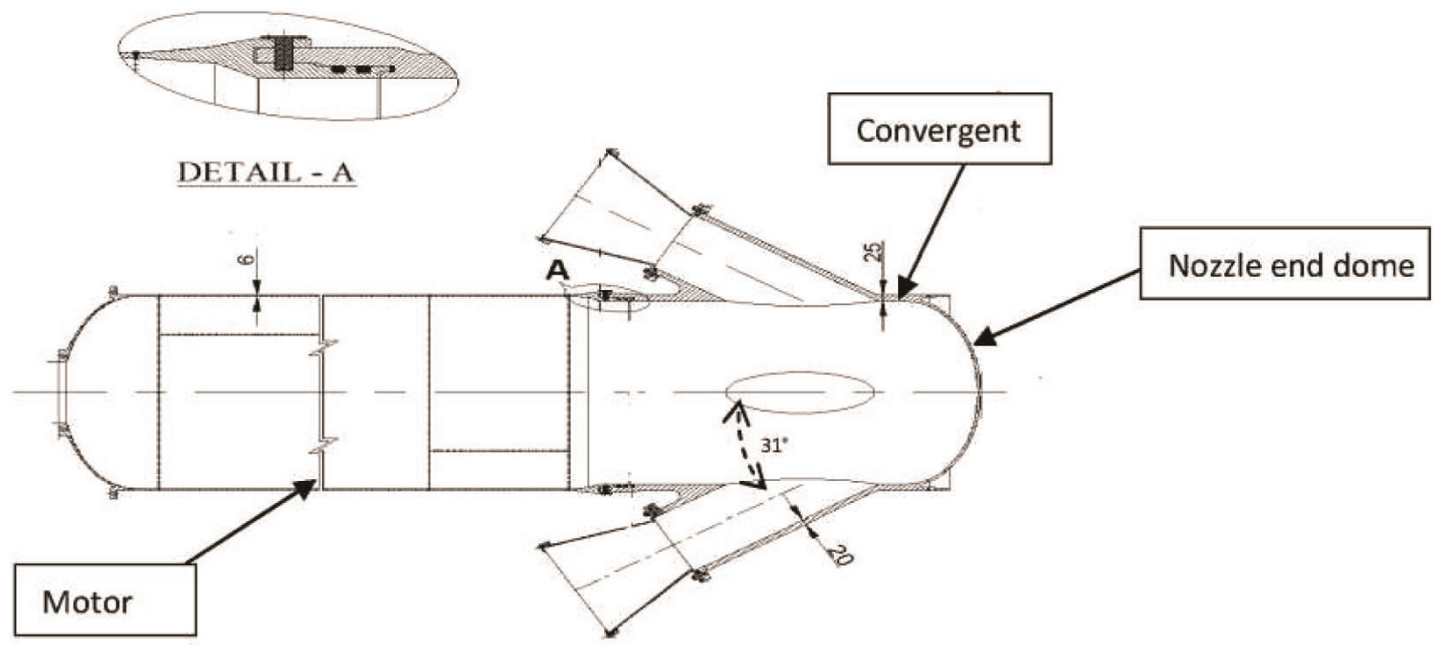

Figure 3. LEM and nozzle convergent assembly.

Box 1. Achieved angular orientation on realized nozzle convergent hardware

Hardware identification

NC-01

NC-02

NC-03

Angular orientation (spec. $31^{\circ \pm 5^{\prime}}$ )

$30^{\circ} 59^{\prime} 36^{\prime \prime} / 31^{\circ} 0^{\prime} 26^{\prime \prime}$

$30^{\circ} 59^{\prime} 38^{\prime \prime} / 31^{\circ} 0^{\prime} 14^{\prime \prime}$

$30^{\circ} 59^{\prime} 52^{\prime \prime} / 31^{\circ} 0^{\prime} 14^{\prime \prime}$

accounting for final machining and weld distortion. OD profile was inspected with template before welding and found to be satisfactory. Control of root gap and fixturing was done during welding to minimize weld distortion. Subsequently, internal machining was done after welding to remove $3 \mathrm{~mm}$ extra stock from nozzle end dome and inspected with ID checking template to ensure profile. Thickness requirement was also ensured during machining. Suiting of the internal ablative liner for first-off hardware was done and the process was found to be satisfactory.

\section{Segment pin hole drilling of nozzle convergent interface}

LEM to nozzle interface is through tongue and groove rings provided with radial holes in which shear pins are put to hold them together (Figure 3). Stringent clearance fit $(16-52 \mu \mathrm{m})$ between shear pins and holes and alignment of pin holes on tongue ring (TR) and groove ring (GR), are the prime requirements for a good assembly. A shear pin hole drill jig was realized (Figure 6) and qualified by mock-up drilling and trial suiting a mild steel version TR and GR simulating the joint. NC hardware tongue ring pinhole drilling was done on $\mathrm{CNC}$ horizontal boring machine using qualified drill jig. During drill jig set-up, drilling and reaming, critical requirements like face butting within $0.05 \mathrm{~mm}$ to control axial alignment of the holes and intermediate checking of pinhole diameters with GO/NOGO gauges were followed. First off NC hardware was successfully realized and smooth assembly was observed with LEM.

\section{Realization and first-off qualification of HEM scarfed divergent aft end hardware}

HEM is one among the four solid motors in CES. HEM nozzle divergent aft end hardware has a scarfed configuration accommodated inside the crew module fairing (CMF) envelope (Figure 7). According to design requirement, divergent aft end hardware inside profile total indicated reading (TIR) shall be within $0.5 \mathrm{~mm}$ along the axis to permit bonding of ablative liner inside. The maximum clearance between hardware and liner allowed is $0.5 \mathrm{~mm}$. No interference is permitted with respect to theoretical contour of the hardware to allow smooth entry of liner. During developmental phase, it was proposed that divergent aft end hardware can be made through sheet rolling and welding of already available ground sheets available in stock, so that schedule can be accelerated. Fabrication process comprises scarf contour development area marking on plates, trimming, rolling and welding to form the cylindrical region. Subsequently, welding of cylindrical shell with nozzle-end flange and finally, machining of interfaces was planned. However, first hardware realized through plate route was inspected and found to have inside profile maximum clearance up to $3.28 \mathrm{~mm}$ against the specification of $0.50 \mathrm{~mm}$ and maximum interference up to $3.11 \mathrm{~mm}$ against the specification of no interference allowed. Runout was measured 
at every $25 \mathrm{~mm}$ interval and found to be $0.873 \mathrm{~mm}$ (at $75 \mathrm{~mm}$ from end face) and $6.480 \mathrm{~mm}$ (at $500 \mathrm{~mm}$ from end face) against the specification of $0.5 \mathrm{~mm}$.

To get better control of the inside profile at final product level, process modification was proposed from plate route to forging route. The flange portion and cylindrical portions of the hardware were planned from two separate forgings, thus reducing material wastage and mass as well as minimum amount of machining. Both forgings were individually heat-treated and then welded. Finally, after nozzle-end flange machining, $30^{\circ}$ scarfing was done on the cylindrical shell by wire-cutting. Inner profile of first hardware realized by forging route was inspected by $\mathrm{CMM}$ and found with a maximum clearance up to
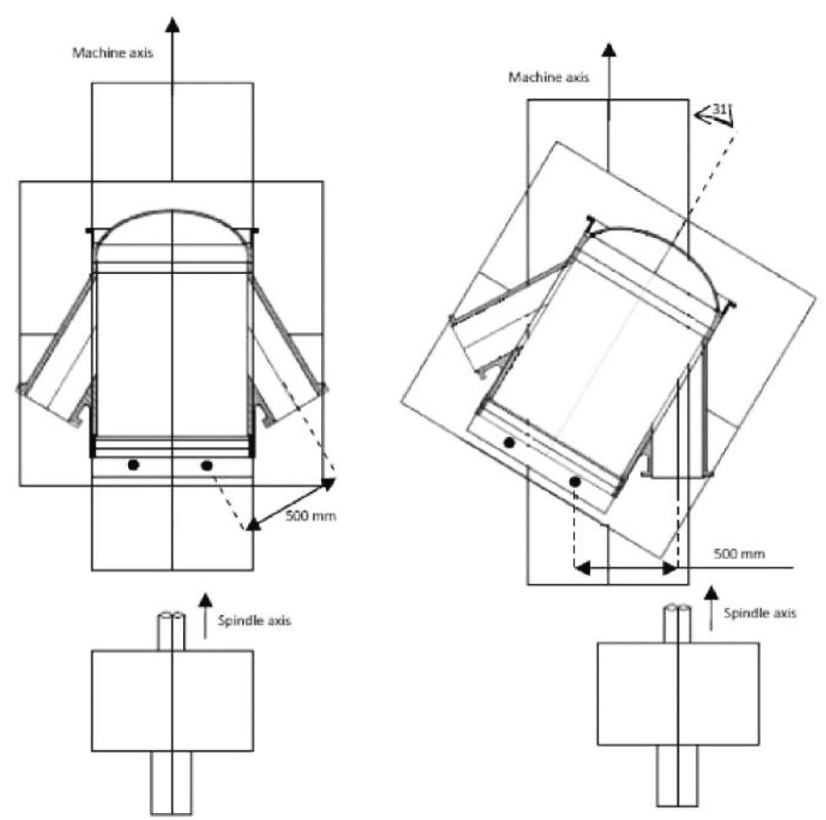

Figure 4. Nozzle boss boring set-up.

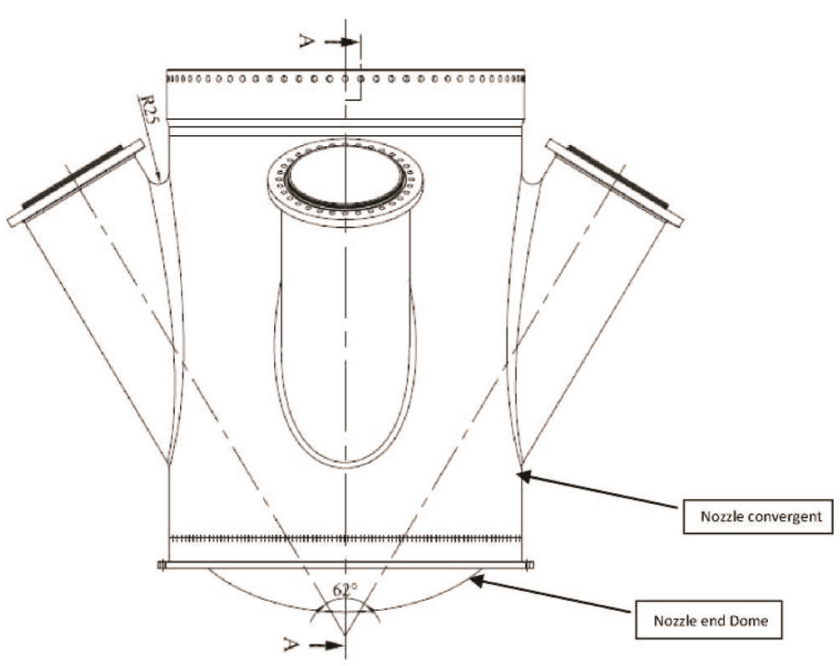

Figure 5. LEM nozzle convergent hardware.
$0.25 \mathrm{~mm}$ and maximum interference up to $0.45 \mathrm{~mm}$. Runout was measured at every $25 \mathrm{~mm}$ interval and found to be $0.18 \mathrm{~mm}$ (at $75 \mathrm{~mm}$ from end face) and $0.53 \mathrm{~mm}$ (at $800 \mathrm{~mm}$ from end face), which was accepted based on satisfactory liner suiting. The above process modification was implemented for subsequent hardware. Four numbers of nozzle divergent hardware were realized by this process and successfully used in PAT.

\section{Occurrence of high strains in CES motors during PPT due to profile error}

As part of the hardware acceptance protocols, the hardware assembly of CES motor to nozzle was subjected to PPT. During PPT of the first CJM case and NC, high strain was observed near the long seam. Maximum hoop strain measured was $7844 \mu$ s on the long seam weld location against the predicted strain of $3614 \mu \mathrm{s}$. Residual strain measured was $1379 \mu$ s. A detailed study performed in the case of first motor revealed that the profile variation near long seam welded joint has contributed to higher strain. Profile variation was due to edge bending near the long seam weld edge, which was not corrected during shell re-rolling operation at post-weld stage, as re-rolling was not recommended over weld reinforcement area. However, LEM case of same material (15CDV6) with similar diameter $(\varnothing 788 \mathrm{~mm})$ and thickness $(6 \mathrm{~mm})$ realized by a different process showed measured strain matching with that predicted during PPT. In this case, the shell was rolled keeping higher length than the required development length for the cylindrical shell so that the edges overlap after rolling. The edges to be welded were subsequently trimmed to correct length. This process gives a nearly circular profile without error near the long seam weld area. Hence the process is revised accordingly for future motor cases.

\section{Conclusion}

Challenges were faced during developmental phase of CES motors due to their inherent configuration and process-related fabrication issues. The processes were specifically designed to achieve stringent requirements in

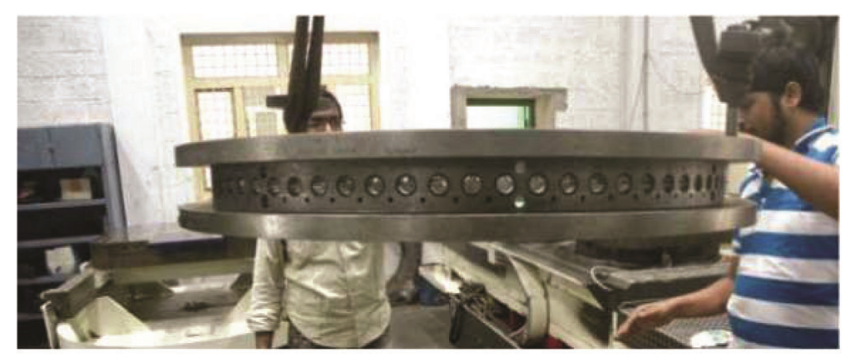

Figure 6. Drill jig for LEM motor case and Nozzle hardware. 


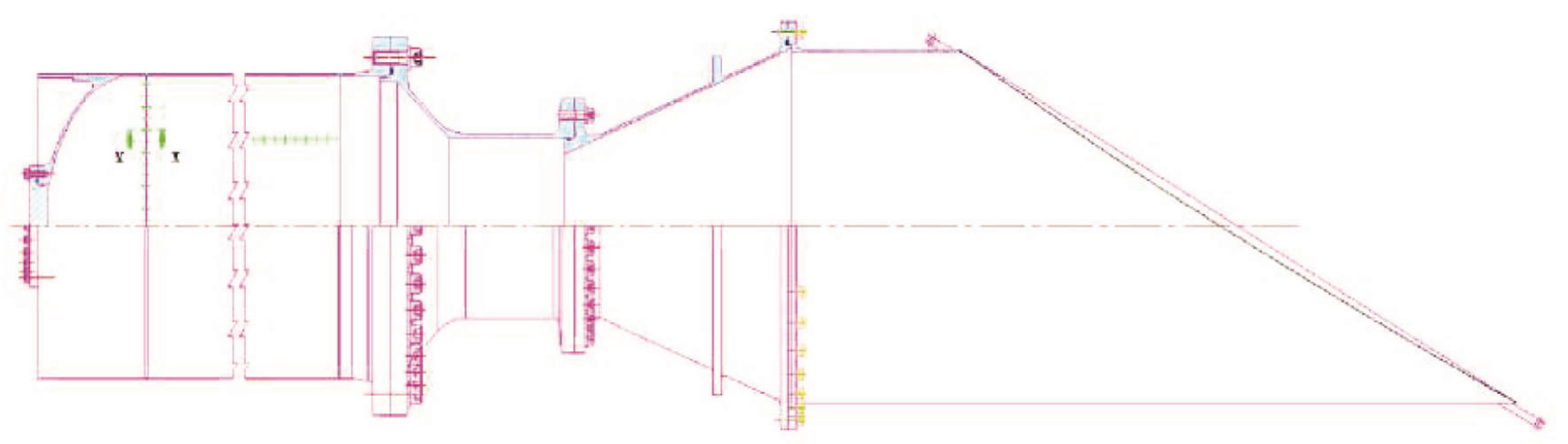

Figure 7. HEM with nozzle convergent and divergent assembly.

geometrical and dimensional accuracies. The issues faced were critically analysed, root cause of the problems identified and possible feasible solution implemented during actual hardware fabrication. After first-off qualification of the hardware, fabrication process and inspection requirements were frozen, constantly monitoring for future hardware fabrication for its conformity. Critical hardware with demanding requirements could be achieved through strict process compliance, mandatory stage clearances and adopting first-off qualification philosophy. Quality Assurance Certification was provided for all CES motors and nozzle hardware identified for static test and PAT, and successfully used.

doi: $10.18520 / \mathrm{cs} / \mathrm{v} 120 / \mathrm{i} 1 / 147-151$ 